\title{
Tratamiento quirúrgico de la fibrilación auricular
}

\section{Surgical treatment of atrial fibrillation}

\author{
Sergio Franco-Sierra ${ }^{a, b, c}$ \\ a Centro Cardiopulmonar y Vascular periférico, Centros Especializados de San Vicente Fundación, Medellín, Colombia \\ b Cirugía Cardiovascular, Clínica Medellín, Medellín, Colombia \\ c Postgrado Cirugía Cardiovascular, Universidad CES, Medellín, Colombia
}

Recibido el 3 de octubre de 2016; aceptado el 6 de octubre de 2016

Disponible en Internet el 11 de noviembre de 2016

\section{Introducción}

La fibrilación auricular es el trastorno del ritmo cardiaco más común y se acompaña de alta morbimortalidad. La presencia de esta patología se convierte en un factor predictor independiente de mortalidad, con un riesgo relativo de mortalidad de 1,5 para hombres y 1,9 para mujeres, además de ser factor predictor independiente para desarrollar ataque cerebrovascular; se ha estimado que el $15 \%$ de las causas de este último son secundarias a fibrilación auricular. El tratamiento médico farmacológico aislado de la fibrilación auricular es complejo, costoso, insatisfactorio e incompleto, características que motivan el interés por el desarrollo y la aplicación de tratamientos alternativos para pacientes con fibrilación auricular ${ }^{1}$. Gracias al adelanto de las técnicas quirúrgicas en el tratamiento de la fibrilación atrial, desarrolladas por el Dr. James Cox y conocidas como cirugía de laberinto o cirugía de "maze", en donde los resultados muestran una curación cercana al $95 \%$, se han desarrollado técnicas quirúrgicas alternativas y reproducibles por la mayoría de los grupos alrededor del mundo, en las se usan diferentes fuentes de energía y se combinan con el tratamiento de enfermedades estructurales, especialmente patologías valvulares del lado izquierdo del corazón. Las guías publicadas en 2014 por la Asociación Americana del Corazón, el Colegio Americano de Cardiología y la Sociedad del Ritmo Cardiaco, están de acuerdo en que es

Correo electrónico: sfrancos@une.net.co razonable realizar ablación de fibrilación atrial en pacientes seleccionados que van a otro tipo de cirugía cardíaca, pero reconocen que aún hay datos limitados y por consiguiente lo consideran una recomendación con nivel de evidencia $C^{1,2}$.

\section{Definición}

La fibrilación auricular se caracteriza por una activación rápida e irregular de la aurícula, que lleva a pérdida del ritmo sinusal normal. Durante esta disritmia varias porciones de la pared atrial se disparan a un ritmo de 400 a 600 veces por minuto, sin que todos los estímulos conduzcan su actividad al ventrículo, puesto que la respuesta de la frecuencia ventricular se encuentra determinada por la interacción entre la actividad auricular y la función de filtro del nodo aurículo-ventricular, el nivel del tono vagal y simpático y la presencia de medicaciones que afectan la conducción aurículo-ventricular (tales como beta-bloqueadores, calcioantagonistas no dihidropiridínicos y digitálicos).

De esta manera, puede existir fibrilación auricular con respuesta ventricular rápida o lenta, según la cual se darán las manifestaciones clínicas y los síntomas derivados de la frecuencia ventricular y la consecuente alteración del estado hemodinámico. La fibrilación auricular en el electrocardiograma se caracteriza por la ausencia de ondas $\mathrm{P}$ claramente definidas, que son reemplazadas por oscilaciones rápidas (ondas fibrilatorias); estas ondas pueden variar en tamaño, forma y frecuencia y se asocian con una respuesta ventricular irregular cuando la conducción AV es normal (fig. 1). 


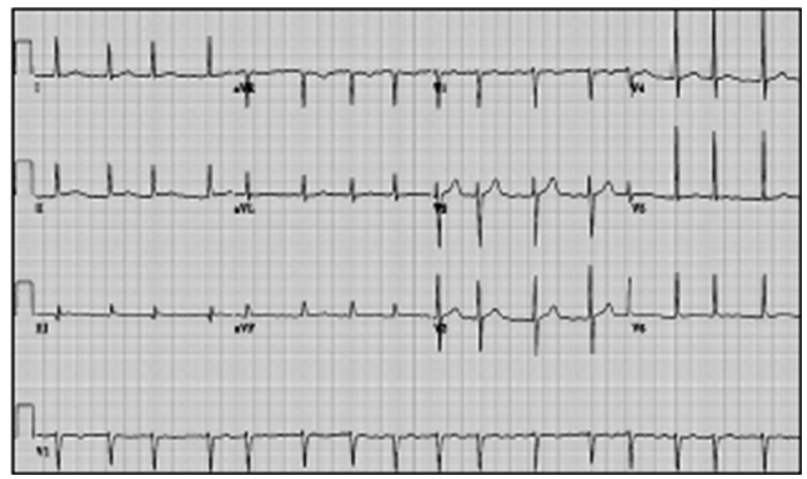

Figura 1 Electrocardiograma de un paciente en ritmo de fibrilación auricular. Se aprecia la ausencia de ondas p y la presencia de un RR variable.

\section{Incidencia}

La fibrilación auricular se presenta aproximadamente en 2,2 millones de personas en los Estados Unidos; su incidencia aumenta con la edad, con una prevalencia de $0,5 \%$ en la quinta década de la vida, se eleva entre el 2-5\% alrededor de los 60 años y asciende hasta el 10\% alrededor de la octava década. La incidencia real en Colombia se desconoce, pero es claro que su presentación cada vez más frecuente, obedece al mayor envejecimiento de la población, mayores comorbilidades asociadas y mejores métodos de diagnóstico. A su vez, la presencia de ataque cerebro-vascular aumenta con la edad en quienes tienen fibrilación auricular; su incidencia oscila alrededor del $1,5 \%$ en la década de los cincuenta y asciende hasta el $23,5 \%$ en la octava. La fibrilación auricular se asocia con patologías cardiacas, las cuales, a su vez, constituyen factores que predisponen su desarrollo; entre estos se incluyen: enfermedad arterial coronaria, enfermedad valvular cardiaca, falla cardiaca congestiva e hipertensión arterial. Se sabe que entre el 40 y el $60 \%$ de los pacientes con enfermedad estructural de la válvula mitral presentan fibrilación auricular, la cual generalmente es permanente ${ }^{3}$.

La corrección aislada de la valvulopatía mitral no afecta de manera sustancial el ritmo preoperatorio, pues la arritmia se conserva hasta en el $75 \%$ de los pacientes llevados a cirugía de la válvula en quienes se presentaba fibrilación atrial desde el preoperatorio y no se realizó ningún procedimiento de ablación adicional. Hasta un 30\% de los casos con fibrilación auricular no tienen patología cardiovascular asociada con la alteración del ritmo cardiaco y esta ocurre de manera aislada como única manifestación de la enfermedad cardiovascular, de allí el nombre de fibrilación atrial aislada. La edad avanzada, el tabaquismo, los desórdenes metabólicos (diabetes y alteraciones tiroideas), entre otros, son causa desencadenante de fibrilación auricular.

La terapia médica farmacológica con antiarrítmicos a largo plazo, se relaciona con una falla en la recuperación del ritmo sinusal del $50 \%$ en el primer año y es cercana al $85 \%$ a los dos años de tratamiento continuo. Adicional a la falla terapéutica, los antiarrítmicos disponibles en la actualidad son tóxicos a largo plazo, no son específicos para la mejoría de la actividad atrial y por tanto pueden generar efectos colaterales adversos sobre la electrofisiología ventricular.
Por ende, el tratamiento médico actual de la fibrilación auricular está enfocado en el control de la respuesta ventricular y el manejo del riesgo de tromboembolia mediante el uso de anticoagulantes orales de por vida (warfarina o anticoagulantes orales directos, según sea la etiología). Está claro que la terapia con warfarina ha demostrado tener un efecto benéfico en la reducción de los eventos tromboembólicos en los pacientes con fibrilación auricular, sin embargo, este tratamiento expone a un riesgo significativo de eventos hemorrágicos, que oscilan alrededor del $3 \%$ por paciente por año ${ }^{4}$.

\section{Clasificación}

La terminología propuesta para la categorización de la fibrilación auricular se aplica a episodios de más de 30 segundos. Es importante definir si se está ante el primer episodio detectado, que recibirá el nombre de evento inicial, bien sea sintomático o no, o autolimitado o no. Si el paciente ha tenido dos o más episodios, se habla de fibrilación auricular recurrente, que a su vez se clasifica en paroxística, persistente o permanente.

De acuerdo con las guías de la Heart Rythm Society 2012, la fibrilación auricular persistente se define como episodios de fibrilación no autolimitados que duran más de 7 días, o menos de 7 días en caso de requerir cardioversión, definición que fue revisada y actualizada en 2014 por el grupo de manejo de ritmo cardíaco de las sociedades científicas ACC-AHA-HRS, definiendo simplemente como fibrilación auricular continua aquella que dura más de 7 días y como persistente (long-standing persistent atrial fibrillation) y continua, aquella que dura más de 12 meses $^{1}$.

Desde el punto de vista patológico, hemodinámico y de valoración terapéutica, la fibrilación auricular de origen valvular, es decir aquella que se presenta de manera concomitante con patología valvular (especialmente mitral), requiere un enfoque diagnóstico y terapéutico diferente, pues su tratamiento es más complejo y la respuesta al manejo usualmente es menos satisfactoria que en la fibrilación auricular no valvular. En ocasiones fue clasificada como taquicardiomiopatía de origen valvular, en cuyo caso la efectividad del procedimiento quirúrgico de ablación, se reduce sustancialmente, en especial cuando se acompaña de dilatación marcada de la aurícula izquierda, factor considerado como de mal pronóstico en la resolución de la fibrilación auricular, cuando el tamaño atrial izquierdo sobrepasa los $5,5 \mathrm{~cm}$. De hecho, las guías de manejo de fibrilación auricular diferencian en cuanto a la necesidad de anticoagulación, según la etiología entre fibrilación auricular valvular y no valvular.

\section{Resultados del tratamiento quirúrgico}

La falla en la respuesta al tratamiento médico farmacológico ha impulsado a los grupos quirúrgicos a desarrollar diferentes técnicas en el tratamiento no farmacológico de esta entidad. El trabajo de Cox et al. demostró la posibilidad de realizar un tratamiento quirúrgico exitoso de la fibrilación auricular, mediante la interrupción de los múltiples circuitos auriculares de reentrada, uno de los principales mecanismos fisiopatológicos propuestos como causa de fibri- 


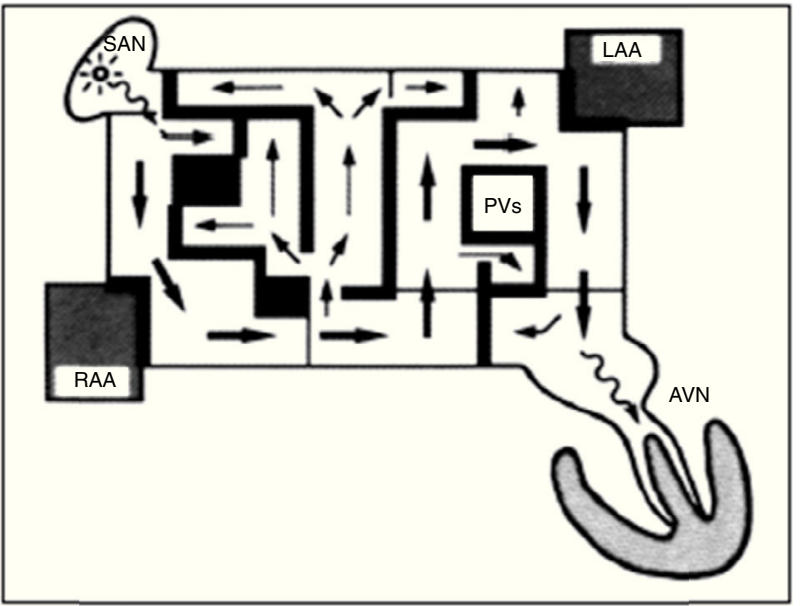

Figura 2 Esquema de la cirugía de laberinto para la técnica de corte y sutura ("Maze").

lación auricular. Con esta técnica quirúrgica, conocida como cirugía de laberinto (Maze), realizada a través de la técnica de corte y sutura, combinada con segmentos de crioablación, se ha logrado curar la fibrilación auricular hasta en el $95 \%$ de los casos (figs. 2 y 3$)^{5-7}$. Infortunadamente, el uso de esta técnica no se ha expandido lo suficiente en el mundo debido a su complejidad y a que no es fácilmente reproducible y aumenta significativamente el tiempo del bypass cardiopulmonar. La investigación actual se enfoca

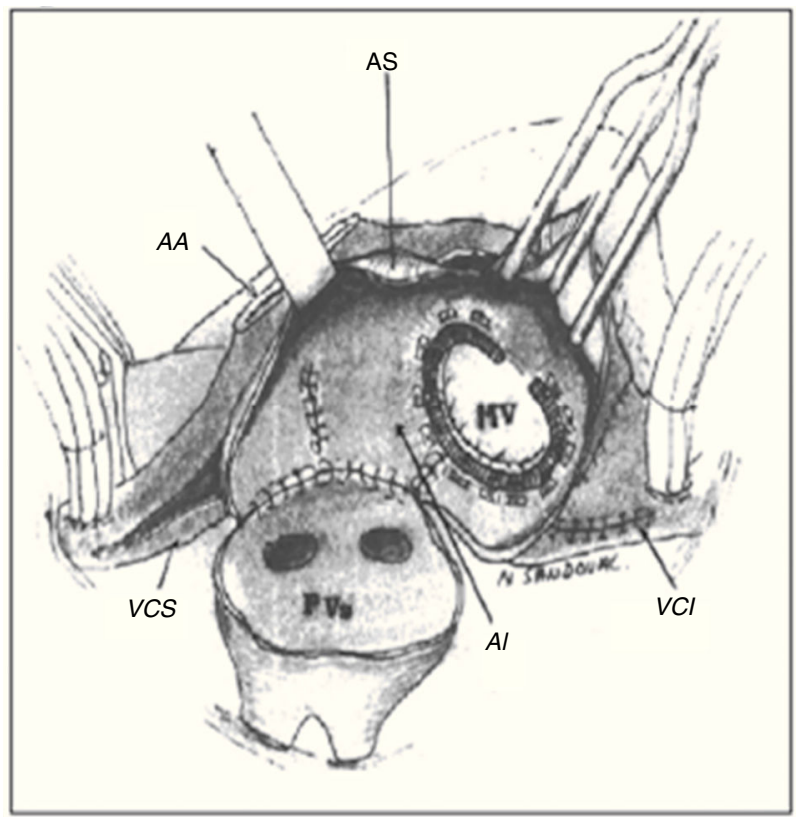

Figura 3 Cirugía de maze III. Representación esquemática de la cirugía de laberinto (Maze). Se aprecia la canulación bicava, las incisiones en vena cava superior e inferior (VCS, VCl), la sección del apéndice atrial derecho (AA), la incisión del septum interauricular (As), la aurícula izquierda (Al) y la válvula mitral, sobre la que se ha colocado un anillo (MV). Adaptada de: Sandoval N., Tratamiento quirúrgico de la fibrilación auricular. En: Franco, S. (Ed). Enfermedad Valvular Cardiaca, 1. ${ }^{a}$ ed. 2004. p. 289. hacia el desarrollo de técnicas más simples, menos invasivas, que requieran menos tiempo adicional de circulación extracorpórea o que puedan obviar esta última y por incisiones mínimas. En simultánea, se han desarrollado fuentes alternas de energía que permiten crear los patrones de lesión auricular de manera rápida, segura y efectiva y que no implican la técnica de corte y sutura.

Los tres objetivos fundamentales durante la cirugía de la fibrilación auricular son:

1. Ablación de la arritmia a través de la abolición de los circuitos de reentrada.

2. Restauración de la sincronicidad aurículo-ventricular, que favorece la conducción a través de las aurículas y el impulso desde el nodo sinusal al nodo AV.

3. Recuperación de la contracción auricular efectiva.

La cirugía de maze ha sufrido cambios a través de los años; el maze ıv, tal como es concebido en la actualidad se considera como el "estándar de oro" en el tratamiento quirúrgico de la fibrilación auricular, con una tasa de curación cercana al $98 \%$ en manos experimentadas (fig. 3, tabla 1). El procedimiento de maze o cirugía de laberinto para fibrilación auricular, asociado a cirugía valvular mitral, ha demostrado excelentes resultados en la curación de la fibrilación auricular. Sus beneficios a largo plazo son óptimos en cuanto a la recuperación de la actividad atrial y el mantenimiento del ritmo sinusal. Con esta técnica se conserva la función atrial izquierda y derecha en cerca del $90 \%$ de los pacientes $^{7}$.

En un intento por realizar un procedimiento más simple, que consuma menos tiempo y que potencialmente disminuya la necesidad de bypass cardiopulmonar, se han desarrollado los procedimientos maze parcial, que generalmente se practican sobre la aurícula izquierda, donde el foco principal de tratamiento está dirigido al aislamiento de las cuatro venas pulmonares y del apéndice atrial izquierdo. En estos intentos se han desarrollado varias fuentes de energía, las cuales pretenden disminuir el tiempo operatorio y generar lesiones transmurales que curen la fibrilación auricular. Se presentan resultados de la técnica de maze parcial, con el porcentaje de éxito en la recuperación del ritmo sinusal (tabla 2).

Los resultados tempranos de estas series han generado un avance muy importante en el desarrollo de la cirugía para fibrilación atrial. Mediante el uso de diversas fuentes de energía, se ha demostrado la posibilidad de efectuar procedimientos menos complejos, que sean fácilmente reproducibles y que además se pueden combinar con procedimientos quirúrgicos para el tratamiento de enfermedad estructural cardiaca. Estos resultados se han conseguido con un incremento mínimo en el tiempo total de circulación extracorpórea y con baja frecuencia de complicaciones relacionadas. Los resultados a corto y largo plazo estimulan a continuar con la técnica, pues se obtiene un porcentaje de éxito variable, entre el 60 - $80 \%$ en la curación de la fibrilación atrial. La fuente ideal de energía deberá ser rápida, de fácil disponibilidad, que genere lesión transmural, que no dañe los tejidos adyacentes y que pueda llegar a ser usada sin circulación extracorpórea y a través de cirugía mínimamente invasiva ${ }^{8-10}$. 
Tabla 1 Resultados de la cirugía de maze III

\begin{tabular}{|c|c|c|c|c|c|c|c|c|c|}
\hline Autor & Año & Seguim & No. Pac. & Maze III & Cx A\% & SIN\% & FAD & FAI & MORT \\
\hline Cox & 1996 & $3 M-8.5 A$ & 178 & 118 & $17 \%$ & $93-10$ & $94 \%$ & $98 \%$ & $2,2 \%$ \\
\hline Cox & 1998 & $3 M-10 A$ & 201 & 155 & $16 \%$ & $96 \%$ & $93 \%$ & $99 \%$ & $2,2 \%$ \\
\hline Sandoval & 1996 & $3 A$ & 21 & 21 & $90 \%$ & $90 \%$ & $71 \%$ & $89 \%$ & $4,3 \%$ \\
\hline Kosakai $^{\mathrm{a}}$ & 1995 & $2 \mathrm{~A}$ & 101 & 101 & $100 \%$ & $84 \%$ & $72 \%$ & $80 \%$ & $2,0 \%$ \\
\hline Kobayashi $^{\mathrm{b}}$ & 1998 & $5 \mathrm{~A}$ & $220 / 61$ & 61 & $100 \%$ & $90 \%$ & & & $1,8 \%$ \\
\hline $\operatorname{Ad~} N^{c}$ & 2002 & $4 \mathrm{~A}$ & 53 & 53 & $100 \%$ & $98 \%$ & & & $3,8 \%$ \\
\hline Bando & 2002 & $7 A$ & 258 & 258 & $100 \%$ & $85 \%$ & & & $2,0 \%$ \\
\hline
\end{tabular}

Adaptada de: Sandoval N. Tratamiento quirúrgico de la fibrilación auricular. En: Franco, S.(Ed) Enfermedad Valvular Cardiaca, $1 .^{\mathrm{a}}$ ed. 2004 p. 289.

Se nota que cuando la cirugía de Maze es usada en el $100 \%$ de pacientes con enfermedad mitral, las posibilidades de curación disminuyen. La Serie de Kobayashi en pacientes seleccionados muestra el $90 \%$ de curación, en grupo seleccionado.

CX A: Cirugía asociada. SIN: Sinusal. FAD: Función aurícula derecha. FAI: Función aurícula izquierda. MORT: Mortalidad.

a No seleccionados

b Seleccionados

c FA paroxística y crónica

Tabla 2 Porcentaje de recuperación del ritmo sinusal según autor y fuente de energía usada

\begin{tabular}{lllll}
\hline Autor & año & $\mathrm{n}$ & Tipo de Lesión & Porcentaje de recuperación ritmo sinusal \\
\hline Kondo & 2003 & 31 & Crioablación, RF & 79,3 \\
Kress & 2002 & 23 & RF & 86 \\
Wellens & 2002 & 30 & RF & 65 \\
Guden & 2002 & 23 & RF & 81 \\
Benussi & 2002 & 132 & RF & 77 \\
Deneke & 2002 & 21 & RF & 82 \\
Mohr & 2002 & 234 & RF & 81,1 \\
Knaut & 2002 & 105 & Microondas & 61 \\
Pasic & 2001 & 48 & RF & 92 \\
\hline
\end{tabular}

RF: radiofrecuencia.

\section{Fuentes de energía}

\section{Radiofrecuencia}

La radiofrecuencia usa una fuente de energía que alterna entre $350 \mathrm{kHz}$ y $1 \mathrm{MHz}$, cuyo fin es generar una lesión térmica. En la actualidad existe suficiente experiencia acumulada gracias a las técnicas de ablación percutánea que usan radiofrecuencia en el tratamiento de las arritmias cardiacas. Este conocimiento ha llevado a desarrollar varios catéteres de energía de radiofrecuencia que son útiles en la cirugía cardiaca abierta, especialmente en combinación con procedimientos sobre la válvula mitral. Estos dispositivos pueden emplearse en el endocardio o en el epicardio y permiten ser colocados de manera directa sobre el sitio deseado, con el fin de crear lesiones transmurales que bloqueen los estímulos eléctricos anómalos.

La mayoría de los procedimientos de ablación por radiofrecuencia utilizan sistemas unipolares o monopolares que se combinan con irrigación dirigida, con el fin de aumentar la penetrabilidad al tejido y disminuir el daño en los tejidos vecinos. En estos sistemas el catéter entra en contacto con el tejido atrial y la energía es liberada durante un tiempo definido y con un recorrido fácilmente determinado y controlado por el cirujano. Los sistemas unipolares poseen una cantidad de limitaciones que están en relación con la naturaleza de la energía que se libera y la poca posibilidad de generar una lesión transmural (objetivo final de la ablación). La temperatura local puede exceder 100 grados centígrados, lo cual lleva a daño local del endotelio, que de algún modo pudiera convertir la superficie en trombogénica. El calor puede ser conducido a través de tejidos vecinos y generar daño de las estructuras vecinas a distancia (esófago y arterias coronarias). Se han descrito lesiones esofágicas con el uso de la radiofrecuencia seca, algo que no sucede con los dispositivos de radiofrecuencia irrigada, en los que la temperatura que se irradia así como la irradiación del calor disminuyen de manera sustancial y por tanto se minimiza la posibilidad de daño de estructuras adyacentes. La aplicación de la energía deberá ser uniforme, constante y continua, con el fin de crear lesiones definidas que no dejen espacio de tejido sano entre ellas.

Los dispositivos bipolares liberan energía de manera más constante y evitan la transmisión de calor a los tejidos vecinos. Estos mismos dispositivos pueden usarse en el epicardio y en pacientes con fibrilación auricular aislada o que son sometidos a cirugía cardiaca sin necesidad de circulación extracorpórea, a través de la aplicación de estos en las cuatro venas pulmonares durante cirugía de revascularización miocárdica con técnica fuera de circulación extracorpórea.

Uno de estos dispositivos, disponible en nuestro medio, con el cual se puede graduar la liberación de la energía 


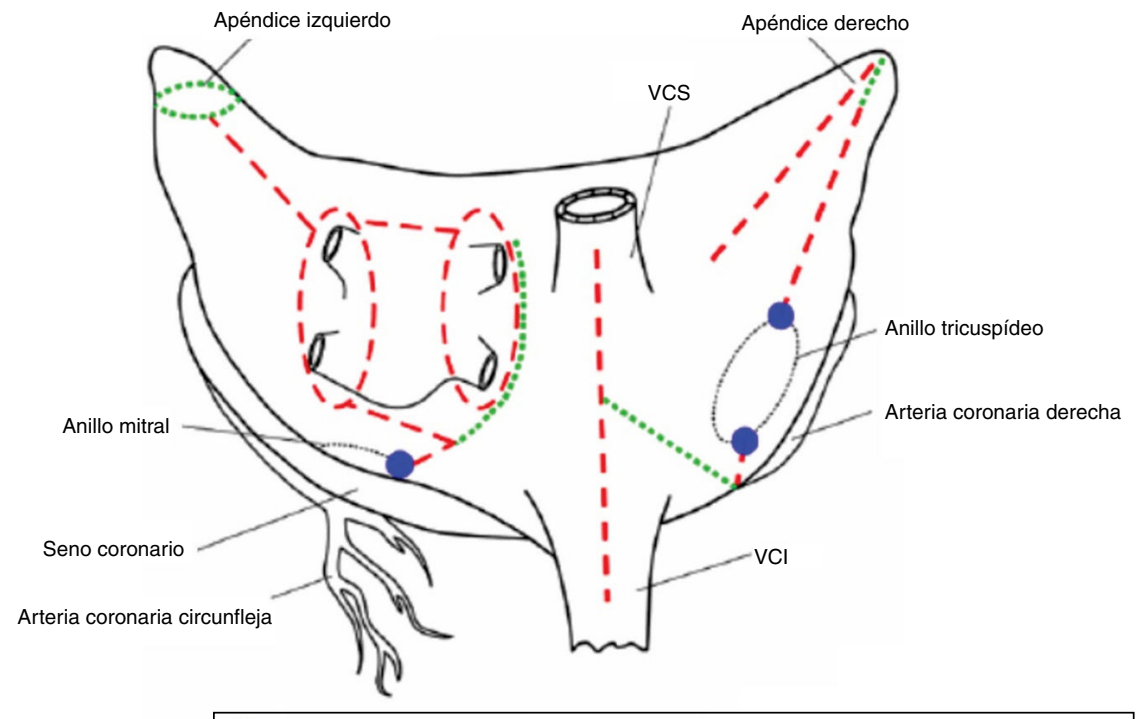

Crioablación .......... Incisión quirúrgica

Figura 4 Líneas de radiofrecuencia. Técnica biatrial de maze ıv, derecho e izquierdo, en la que se combina corte, sutura y radiofrecuencia bipolar irrigada.

según la densidad del tejido, utiliza un catéter de ablación a través de un sistema monopolar irrigado, el cual tiene la ventaja de evitar la transmisión del calor a las estructuras vecinas, impedir el daño de tejidos circundantes y generar transmuralidad gracias a que su sistema de irrigación controlado disminuye el calor y la impedancia y por consiguiente, permite una mayor penetración al tejido. Este dispositivo es maleable, con lo cual se accede fácilmente a las cavidades cardiacas. La máquina viene con un contador de tiempo que permite definir la duración de la ablación en cada territorio ${ }^{11-13}$

En casos de cirugía de ablación aislada o combinada con cirugía valvular, se puede usar el sistema bipolar y realizar la ablación con el uso de circulación extracorpórea o sin esta, rodeando las cuatro venas pulmonares. De la misma forma se puede realizar cirugía de revascularización miocárdica fuera de circulación extracorpórea más ablación epicárdica bipolar sin bomba. En casos de cirugías combinadas, las cuales constituyen la mayoría de procedimientos, la técnica de la ablación se realiza durante el acto operatorio, a través de la misma incisión usada para el reparo o reemplazo de la válvula mitral. En estas cirugías hay dos modalidades, la primera es realizar maze ıv biatrial, siguiendo las líneas de ablación derechas e izquierdas descritas por Cox, y combinar corte y sutura con la radiofrecuencia, tal como se muestra en la fig. 4.

Gracias a los conceptos desarrollados por varios grupos y a la investigación de la generación de fibrilación auricular en el tejido de las venas pulmonares se ha simplificado la técnica y se aplica el concepto de maze parcial o izquierdo, mejor conocido como aislamiento de venas pulmonares, el cual combina diferentes líneas de ablación en la aurícula izquierda, el apéndice atrial, la base de la aurícula y el anillo mitral. La técnica de mayor uso en el momento es el aislamiento de venas pulmonares, que se puede llevar a cabo de manera global rodeando las cuatro venas pulmonares, o parcial haciendo dos bloques independientes para cada segmento de venas pulmonares (derecha e izquierda) Esta técnica disminuye aún más el tiempo requerido para la ablación, sin sacrificar el éxito de los resultados y sin generar morbimortalidad adicional para el paciente (fig. 5).

Con el maze parcial (o izquierdo) se presentan complicaciones como taquiarritmias generadas en el lado derecho, entre las que el flutter atrial es la de mayor importancia; se ha descrito su presencia hasta en el $10 \%$ de los pacientes sometidos a maze izquierdo ${ }^{14,15}$.

\section{Críotermia}

La crioablación se hace con óxido nitroso, el cual se transmite a través de un dispositivo que se aplica a la aurícula a

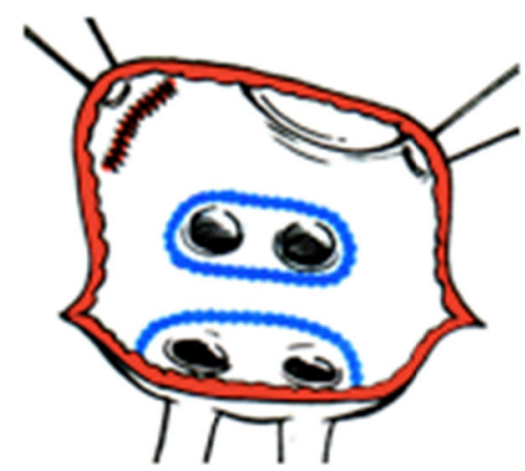

Figura 5 Atriotomía izquierda para la realización de la cirugía de ablación de venas pulmonares. Se aprecian las líneas de ablación, las cuales pueden hacerse de manera individual, rodeando las venas pulmonares derechas e izquierdas en forma aislada, o creando una lesión circunferencial total que abarca las cuatro venas pulmonares (box type lesion). 


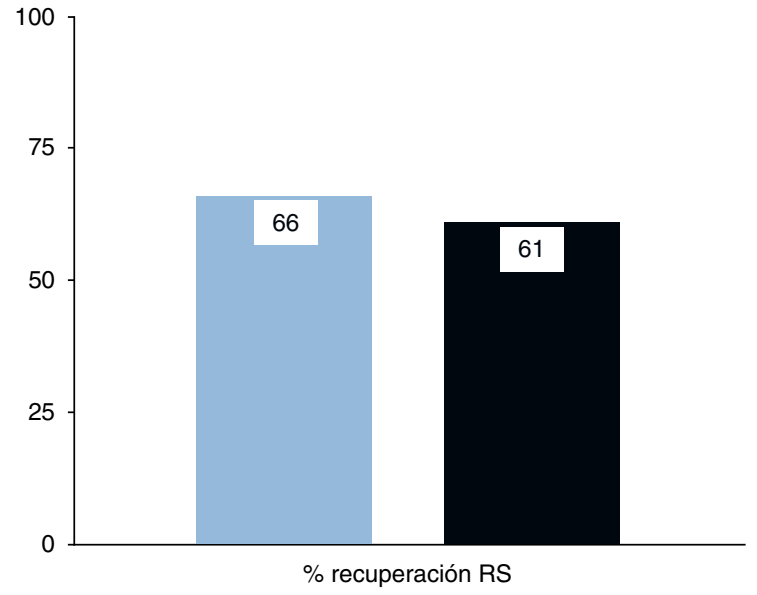

Maze biatrial Aislamiento 4 VP

Figura 6 Porcentaje de recuperación del ritmo sinusal comparando técnica biatrial completa o maze biatrial frente al aislamiento de las cuatro venas pulmonares (4 VP).

una temperatura de menos 60 grados centígrados, durante dos minutos, con el fin de producir lesiones transmurales que bloqueen la conducción atrial. Una de las ventajas de esta técnica es que no existe vaporización del tejido y no hay daño endotelial, ya que después de la aplicación del crío, el tejido permanece liso y sin lesiones macroscópicas evidentes. Cox et al. fueron los primeros en implementar esta técnica en el tratamiento de la cirugía de fibrilación auricular y permanece como un componente muy importante de procedimiento convencional de maze; puede usarse de manera aislada o se puede combinar con técnicas de radiofrecuencia, corte y sutura.

Se han desarrollado y probado otras fuentes de energía como el láser, el ultrasonido de alta intensidad y la radiofrecuencia monopolar, pero los resultados en la ablación de la fibrilación auricular no han sido los esperados y han sido superados de manera amplia con las técnicas de radiofrecuencia bipolar irrigada, razón por la cual han caído en desuso.

Como se mencionó, la técnica más difundida en los grupos quirúrgicos es la ablación de las venas pulmonares, mediante el uso de radiofrecuencia bipolar irrigada o a través de criotermia; la técnica más usada y más simple se basa en la ablación circunferencial de las cuatro venas pulmonares, siempre asociada al cierre de la auriculilla izquierda (con sutura endomiocárdica o con dispositivos epicárdicos de cierre atrial), que se realizan en forma concomitante con el tratamiento de la valvulopatía respectiva. Otros grupos practican la técnica de maze biatrial, en la cual se requiere atriotomía izquierda y derecha, lesiones más complejas con líneas de ablación al anillo mitral y tricuspídeo y generalmente uso de dispositivos combinados, técnica que aumenta significativamente el tiempo de circulación extracorpórea, la posibilidad de complicaciones, el bloqueo AV completo y el costo total del procedimiento. Adicionalmente, según estudios aleatorizados recientes no ha demostrado mayor recuperación del ritmo sinusal a largo plazo, frente a la técnica de ablación de las cuatro venas pulmonares ${ }^{15}$ (fig. 6).

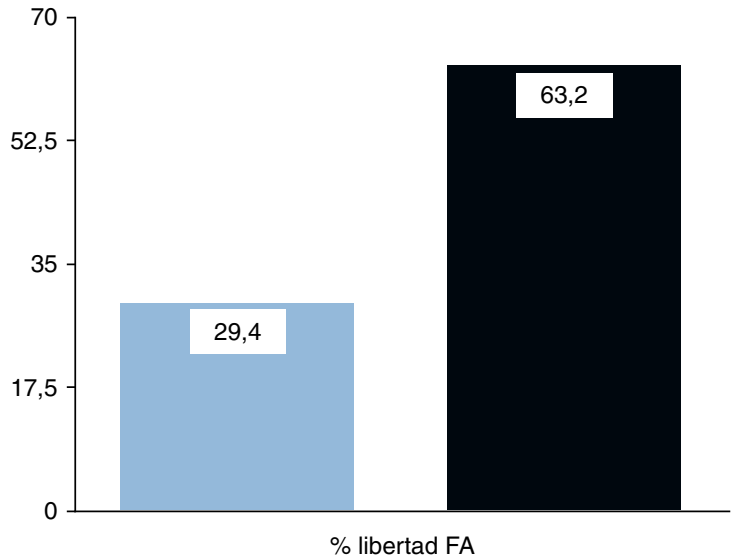

CVM $\square$ CVM + Ablación

Figura 7 Porcentaje de libertad de fibrilación atrial, a un año de seguimiento, cuando se compara cirugía valvular mitral aislada (CVM), frente a CVM más aislamiento de venas pulmonares (CMV + ablación).

En un paciente que se halla en fibrilación auricular permanente, la adición de un procedimiento de ablación durante cirugía valvular mitral, reduce significativamente la fibrilación auricular postquirúrgica. En otras palabras, la posibilidad de recuperar el ritmo sinusal es más del doble cuando se adiciona la técnica de ablación al procedimiento de reparo o reemplazo de la válvula mitral. (fig. 7). Hay estudios que reportan menor incidencia de fibrilación auricular postquirúrgica, pero al parecer no impactan la sobrevida ni los eventos adversos mayores en el seguimiento a un año, mientras que sí se duplica la posibilidad de requerir un marcapasos definitivo. Hoy nuestra conducta quirúrgica es realizar de manera concomitante en el paciente valvular mitral, la cirugía prevista sobre la válvula y la ablación bipolar irrigada, tipo aislamiento de las 4 venas pulmonares, con una tasa de éxito que sobrepasa el $62 \%$ en el seguimiento a 1 año. Se necesita seguimiento a más largo plazo y sistemas de monitorización de larga data (monitorización Holter de frecuencia cardiaca continua de 7 días) que puedan corroborar la persistencia del ritmo sinusal en los pacientes sometidos a ablación quirúrgica ${ }^{16}$.

\section{Conclusiones}

En definitiva, la fibrilación atrial es una patología que atenta contra la vida, tanto como factor predictor independiente de mortalidad como de morbilidad aislada. Por sí misma, puede duplicar el riesgo de muerte en una población de iguales características cuando se comparan individuos con y sin fibrilación auricular. Asociado a esto, la posibilidad de desarrollar eventos tromboembólicos al sistema nervioso central, convierte a la fibrilación auricular en una de las principales causas aisladas de ataque cerebro-vascular; uno de cada seis pacientes con ataque cerebro-vascular tiene como origen de su problema una fibrilación auricular. Aunado a lo anterior, la incidencia de fibrilación auricular 
en la población general, que puede ser del 1\%, asciende hasta el $10 \%$ entre la séptima y octava décadas de la vida, convirtiéndose en un problema de salud pública global. Cuando se presentan anormalidades estructurales del corazón, patología valvular izquierda, enfermedad coronaria o hipertensión arterial esta incidencia es mucho mayor que la reportada en pacientes con corazones estructuralmente sanos. Tal es el caso de los pacientes con enfermedad de la válvula mitral, en quienes la asociación de fibrilación auricular y patología valvular, oscila entre el 50 y el $80 \%$. Todos estos datos hacen que los grupos médicos y quirúrgicos de todo el mundo estén enfocados en el tratamiento, la prevención y la curación de pacientes con fibrilación auricular ${ }^{17}$.

Los avances recientes en el desarrollo de la cirugía de la fibrilación auricular, han permitido contar con procedimientos más rápidos, simples, menos invasivos y con una eficacia similar a la de la cirugía de corte y sutura. Múltiples investigadores han publicado los resultados de la cirugía parcial, sobre el lado izquierdo, utilizando diversas fuentes de energía, con el fin de realizar ablación de las vías anómalas de conducción intrauricular. Estos resultados no han sido comparados a largo plazo con la técnica convencional de maze IV; sin embargo, los resultados obtenidos con las diferentes técnicas a corto y mediano plazo, hacen de esta cirugía y de las diferentes fuentes de energía, herramientas fundamentales y definitivas en el tratamiento de la fibrilación auricular.

De otro lado, algunos de los criterios en la no recuperación del ritmo sinusal bajo tratamiento médico son: tamaño de la aurícula izquierda $>50 \mathrm{~mm}$, duración de la fibrilación auricular mayor a 12 meses, falla cardíaca congestiva (clase funcional NYHA III - IV), función ventricular izquierda $(\mathrm{FE}<30 \%)$ y síndrome del nodo sinusal enfermo, número de cardioversiones previas $(>3)^{18}$. De igual manera se debe conocer que existen criterios clínicos preoperatorios, tales como el aumento del tamaño de la aurícula izquierda ( $>60 \mathrm{~mm})$, el tamaño de la onda $F$ (< $1 \mathrm{~mm})$, la duración o cronicidad de la fibrilación auricular (> 10 años), el índice cardiotorácico (mayor de 70\%), la edad avanzada (mayor de 70 años) y la enfermedad coronaria concomitante, que son predictores en la falla de la recuperación del ritmo sinusal luego de la cirugía antiarrítmica.

Los futuros avances en el entendimiento de la fisiopatología de la fibrilación auricular, así como en la selección individual de pacientes a través de técnicas de mapeo intraauricular avanzadas, permitirán hacer una mejor selección de pacientes y optimizar los porcentajes de curación de la fibrilación auricular. La utilidad de fuentes de energía que facilitan la técnica de maze parcial o el aislamiento de las cuatro venas pulmonares, está bien documentada; es una técnica segura y eficaz y permite adicionar esta cirugía en procedimientos concomitantes que impliquen el reemplazo o preparo de la válvula mitral, con tan solo 10 a 20 minutos en promedio de tiempo extra de pinzamiento aórtico y circulación extracorpórea. Estas tecnologías admiten en la actualidad realizar estos mismos procedimientos con el corazón batiendo y por vía epicárdica, e igualmente, en el momento actual, pueden llevarse a cabo a través de pequeñas incisiones.

\section{Conflicto de intereses}

Los autores declaran no tener conflicto de intereses.

\section{Bibliografía}

1. January CT, Wann LS, Alpert JS, et al. 2014 AHA/ACC/HRS guideline for the management of patients with atrial fibrillation: executive summary: a report of the American College of Cardiology/American Heart Association Task Force on practice guidelines and the Heart Rhythm Society. Circulation. 2014;130:2071-104.

2. Calkins H, Kuck KH, Cappato R, et al. 2012 HRS/EHRA/ECAS Expert Consensus Statement on Catheter and Surgical Ablation of Atrial Fibrillation: recommendations for patient selection, procedural techniques, patient management and follow-up, definitions, endpoints, and research trial design. Europace. 2012;14:528-606.

3. Gillinov AM, Blackstone EH, McCarthy PM. Atrial fibrillation: current surgical options and their assessment. Ann Thorac Surg. 2002;74:2210-7.

4. Knaut M, Tugtekin SM, Spitzer S, et al. Combined atrial fibrillation and mitral valve surgery using microwave technology. Semin Thorac Cardiovasc Surg. 2002;14: 226-31.

5. Sundt TM, Camillo CJ, Cox JL. The maze procedure for cure of atrial fibrillation. Cardiol Clin. 1997;15:739-48.

6. Cox JL, Schuessler RB, D'Agostino HJ Jr, et al. The surgical treatment of atrial fibrillation. III. Development of a definitive surgical procedure [comment]. J Thorac Cardiovasc Surg. 1991;101:569-83.

7. McCarthy PM, Gillinov AM, Castle L, et al. The Cox-Maze procedure: the Cleveland Clinic experience. Sem Thorac Cardiovasc Surg. 2000;12:25-9.

8. Benussi S, Nascimbene S, Agricola E, et al. Surgical ablation of atrial fibrillation using the epicardial radiofrequency approach: mid-term results and risk analysis. Ann Thorac Surg. 2002;74:1050-6.

9. Deneke T, Khargi K, Grewe PH, et al. Left atrial versus bi-atrial Maze operation using intraoperatively cooled-tip radiofrequency ablation in patients undergoing open-heart surgery: safety and efficacy. J Am Coll Cardiol. 2002;39: 1644-50.

10. Mohr FW, Fabricius AM, Falk V, et al. Curative treatment of atrial fibrillation with intraoperative radiofrequency ablation: short-term and midterm results. J Thorac Cardiovasc Surg. 2002;123:919-27.

11. Pasic M, Bergs $P$, Muller $P$, et al. Intraoperative radiofrequency maze ablation for atrial fibrillation: the Berlin modification. Ann Thorac Surg. 2001;72:1484-90.

12. Williams MR, Stewart JR, Bolling SF, et al. Surgical treatment of atrial fibrillation using radiofrequency energy. Ann Thorac Surg. 2001;71:1939-43.

13. Sie HT, Beukema WP, Misier AR, et al. Radiofrequency modified maze in patients with atrial fibrillation undergoing concomitant cardiac surgery [comment]. J Thorac Cardiovasc Surg. 2001;122:249-56.

14. Ad N, Henry L, Massimiano P, et al. The state of surgical ablation for atrial fibrillation in patients with mitral valve disease. Curr Opin Cardiol. 2013;28:170-80.

15. Bogachev-Prokophiev A, Zheleznev S, Romanov A, et al. Ablation for atrial fibrillation during mitral valve surgery: 1-year results through continuous subcutaneous monitoring. Interact Cardiovasc Thorac Surg. 2012;15:37-41. 
16. Gillinov M, Gelijns A, Parides M, et al., for the CTNS invetigators. Surgical ablation of atrial fibrillation during mitral valve surgery. N Engl J Med. 2015;372:1399-409.

17. Gillinov M, Soltesz E. Surgical treatment of atrial fibrillation: today's questions and answers. Semin Thorac Cardiovasc Surg. 2013;25:197-205.
18. Lee R, McCarthy PM, Wang EC, et al. Midterm survival in patients treated for atrial fibrillation: a propensity-matched comparison to patients without a history of atrial fibrillation. J Thorac Cardiovasc Surg. 2012;143:1341-51. 